\title{
Revisión de la relación existente entre la exposición ocupacional al formaldehído y leucemia
}

\author{
Review relationship between occupational exposure to formaldehyde and \\ leukemia
}

\author{
Katerine Greace Ajalla Puente ${ }^{1-2-3}$, Claudia Sandoval Polanco 1-2-4, Mónica Nitu 1-2-5, Ana María Sancho Prades ${ }^{1-2-6}$ \\ 1. Unidad Docente de Medicina del Trabajo del País Vasco. Vitoria (Álava). España \\ 2. Escuela Nacional de Medicina del Trabajo. Madrid. España \\ 3. Hospital de Basurto. Bilbao (Vizcaya). España \\ 4. Hospital Universitario de Álava. Vitoria (Álava). España \\ 5. Hospital Universitario de Cruces, Barakaldo (Vizcaya). España \\ 6. Hospital Universitario Donostia. San Sebastián. España
}

Recibido: 27-12-12

Aceptado: 26-02-13

\section{Correspondencia}

Katerine Greace Ajalla Puente

Hospital Basurto. Unidad de Salud Laboral

Avda. de Montevideo, 18

48013 Bilbao (Vizcaya). España.

Tfno.: 944418800

Correo electrónico: katerinegreace.ajallapuente@osakidetza.net

\section{Resumen}

Introducción: El formaldehído es un cancerígeno conocido, su relación con un aumento del riesgo de leucemia en exposición ocupacional continua en debate a pesar de los diversos estudios realizados. Presentamos una revisión bibliográfica cuyo objetivo es conocer el nivel de evidencia existente entre la posible relación causal de la exposición laboral al formaldehído y la aparición de leucemia, mediante el análisis sistemático de la producción científica publicada entre los años 2008 y 2012.

Metodología: Se realizó una búsqueda bibliográfica en las bases de datos Medline, IBECS, LILACS, CROCHRANE, OSH UPDATE, CISDOC, WEB OF KNOWLEDGE y SCOPUS, utilizando una estrategia de búsqueda a partir de términos "MeSH". Se obtuvieron un total 302 artículos de los que finalmente se seleccionaron 7 que cumplían los requisitos establecidos. De ellos, 4 eran metaanálisis, 2 estudios de casos y controles, 1 estudio de cohortes. Para la asignación del nivel de evidencia se utilizaron los criterios de la Scottish Intercollegiate Guidelines Network (SIGN).

Resultados: Se han encontrado riesgos elevados para los niveles de máxima exposición y también datos de mortalidad estadísticamente significativa para leucemia mieloide con aumento del número de años de prácticas de embalsamamiento. Tres metaanálisis aportan RR altos para leucemia mieloide. Un cuarto metaanálisis al excluir los estudios de mortalidad proporcional obtiene que los resultados basados en cohortes y estudios caso-control no sugieren una asociación entre exposición al formaldehído y leucemia.

Discusión: Los estudios disponibles presentan limitaciones que no hacen posible establecer niveles de evidencia suficientes que confirmen la relación concluyente entre exposición a formaldehído y aparición de leucemias en trabajadores. Nuestra revisión bibliográfica contiene estudios heterogéneos en diferentes 
poblaciones donde hemos encontrado valores de asociación (RR, OR) superiores a 1 en algunos estudios y hallazgos de alteraciones cromosómicas en personas expuestas en el ámbito laboral. Estos datos constituyen una base interesante para investigaciones futuras donde la utilización de biomarcadores de dosis interna acumulada (Aductos ADN-Formaldehído, Glutatión-lesión de ADN inducida por formaldehído) podrán verificar mejor esta asociación.

Med Segur Trab (Internet) 2013; 59 (230) 112-123

Palabras Clave: Formaldebído, Leucemia, Exposición ocupacional.

Abstract

Background: Formaldehyde is a known carcinogen, its relationship to an increased risk of leukemia in occupational exposure continues being debated despite several studies. We present a literature review which aim is to know the level of evidence between the possible causal relationship of occupational exposure to formaldehyde and the development of leukemia, through the systematic analysis of the scientific production published from 2008 to 2012.

Methods: We performed a literature search in the databases Medline, IBECS, LILACS, CROCHRANE, OSH UPDATE, CISDOC, WEB OF KNOWLEDGE and SCOPUS, using a search strategy based on terms "MeSH". We obtained a total of 302 items, finally it were selected 7 that met all the requirements. Of these, 4 were meta-analyzes, two case-control studies and 1 cohort study. To assign the level of evidence we used the criteria of the Scottish Intercollegiate Guidelines Network (SIGN).

Results: We found elevated risks for high levels of exposure and mortality data also statistically significant for myeloid leukemia with increased number of years of embalming practices. Three meta-analyzes provide high RR for myeloid leukemia. A fourth meta-analysis by excluding proportional mortality studies that the results obtained based on cohort and case-control studies do not suggest an association between formaldehyde exposure and leukemia.

Discussion: The available studies have limitations that do not make it possible to establish sufficient levels of evidence confirming the conclusive relationship between formaldehyde exposure and the development of leukemia in workers. Our literature review contains heterogeneous studies in different populations ; we found values of association (RR, OR) greater than 1 in some studies and findings of chromosomal abnormalities in exposed individuals at the workplace. These data provide an interesting basis for future research about the use of accumulated internal dose biomarkers (DNA-formaldehyde adducts, Glutathione-induced DNA by formaldehyde) can better verify this association.

Med Segur Trab (Internet) 2013; 59 (230) 112-123

Key words: Formaldehyde, Leukemia, occupational exposure. 


\section{INTRODUCCIÓN}

El formaldehído, tradicionalmente, llamado metanal o aldehído fórmico, es un aldehído que se presenta a temperatura ambiente en forma de gas incoloro de olor acre y sofocante, en solución acuosa se denomina formol o formalina. Es un compuesto inflamable y puede formar mezclas explosivas con aire y oxígeno.

Este compuesto es un producto normal del metabolismo humano y además se desprende durante la pirólisis de muchas materias orgánicas (incineradores, gases de escape de los automóviles, humo de cigarrillos, etc.). Es ampliamente utilizado en la industria, así como en el ámbito sanitario por ser un poderoso antiséptico, germicida, fungicida y preservador ${ }^{1}$.

En el medio laboral, la principal vía de exposición es la inhalatoria, ya que la sustancia es muy volátil y se deposita fácilmente en las vías respiratorias, principalmente en la vía aérea superior. Al utilizarse en disolución acuosa, también existe riesgo de absorción cutánea pero éste tipo de absorción es reducida ${ }^{2}$.

En el ámbito hospitalario el formol se utiliza principalmente para fijación y conservación de muestras de tejidos, conservación de órganos e incluso cadáveres. También se utiliza como esterilizante, en autoclaves específicos y como desinfectante de alto nivel en limpiezas superficiales, junto con otros aldehídos. El personal con más riesgo de exposición es el que trabaja en las salas de embalsamar, anatomía, histología, diálisis, esterilización, desinfección, laboratorio, odontología y farmacia.

Los usos industriales del formaldehído son diversos, incluyendo la producción de adhesivos y aglutinantes para madera, plástico, textiles, cuero y en la fabricación de productos químicos. Cerca de 21 millones de toneladas de formaldehído se producen anualmente en todo el mundo. En el año 2000 la producción de formaldehído en Estados Unidos superó los 4,6 millones de toneladas ${ }^{3}$. Se estima que en la Unión Europea, más de un millón de trabajadores están expuestos de alguna forma al formaldehído ${ }^{4}$.

A nivel mundial existe una gran discordancia entre los límites de exposición profesional al formaldehído (tabla I), incluso dentro de un mismo país, como ocurre entre distintas jurisdicciones de Canadá o entre diferentes organizaciones de referencia de Estados Unidos como pueden ser la Administración de Seguridad y Salud Ocupacional (OSHA), La Conferencia EEUU de Higienista Industriales Gubernamentales (ACGIH) y El Instituto Nacional para la Salud y Seguridad en el Trabajo (NIOSH) ${ }^{2}$.

En la tabla I se presentan los valores límite de exposición profesional admitidos en diferentes Países.

Tabla I. Valores límite de exposición profesional por países

\begin{tabular}{llcc}
\hline \multicolumn{1}{c}{ País u Organización } & $\begin{array}{c}\text { VLA-ED } \\
(\mathrm{ppm})\end{array}$ & $\begin{array}{c}\text { VLA-EC } \\
(\mathrm{ppm})\end{array}$ & $\mathrm{C}$ (ppm) \\
\hline Alemania & 0.3 & 0.6 & 1 \\
Reino Unido & 2 & 2 & - \\
Hungría & 0.5 & 0.5 & - \\
Francia & 0.5 & 1 & - \\
Japón & 0.1 & - & - \\
España & - & 0.3 & - \\
EEUU (OSHA) & 0.75 & 2 & - \\
EEUU (NIOSH) & 0.016 & - & 0.1 \\
EEUU (ACGIH) & - & - & 0.3 \\
\hline
\end{tabular}

VLA-EC: Valor límite ambiental de corta duración.

VLA-ED: Nivel de exposición en 8 horas. 
Se puede comprobar como en ningún caso se toleran valores de exposición superiores a $2 \mathrm{ppm}$. Varios de los organismos de referencia indican, que en dosis inferiores a 1 ppm, los efectos tóxicos del formaldehído en el material genético (efecto genotóxico) sólo juega un papel mínimo. Sin embargo, valores de exposición crónica superiores a los 2 ppm pueden convertirse en un factor de riesgo principal.

En el año 2004 la Agencia Internacional para la Investigación sobre el Cáncer (IARC) reclasificó el formaldehído como un cancerígeno humano que causa el cáncer de la nasofaringe y también llegó a la conclusión de que existe una "fuerte pero no suficiente evidencia para una asociación causal entre la leucemia y la exposición ocupacional a formaldehído", basada en la evidencia de estudios epidemiológicos 5 .

El Instituto Nacional de Seguridad e Higiene del Trabajo dependiente del Ministerio del Trabajo y Asuntos Sociales de España en su página web mantiene su clasificación anterior como probablemente cancerígeno ${ }^{6}$.

Para la leucemia, los datos más fiables sobre incidencia a escala internacional corresponden a la población norteamericana, donde se observa que la incidencia acumulada (todos los tipos de leucemia) es casi el doble que la mortalidad declarada: 20 casos por cien mil habitantes y año (13 casos por cien mil habitantes/año en los hombres y 7,4 en las mujeres $)^{7}$. Se postula que pueda existir una tendencia ascendente en la incidencia de leucemia debido a la aparición de nuevas exposiciones medioambientales, al aumento de casos de SIDA y a los cambios en la estructura poblacional producidos por la migración y el envejecimiento de la población.

Por lo tanto, determinar si existe una asociación entre la exposición al formaldehído y la aparición de leucemia es de gran importancia para la salud pública, sin embargo, la discrepancia de criterios, diversidad de resultados en estudios realizados tanto en humanos como en animales y las dificultades para homogeneizar los valores límites mínimos, se presentan como los principales retos a la hora de evaluar el riesgo de leucemia asociada con la exposición al formaldehído ${ }^{8}$.

El objetivo de nuestra revisión bibliográfica es disponer de la mayor evidencia existente y conocer la posible relación causal entre la exposición al formaldehído y la aparición de leucemia en el ámbito laboral.

\section{MATERIAL Y MÉTODOS}

Se realizó una revisión bibliográfica utilizando como fuentes de información las siguientes bases de datos: MEDLINE, CISDOC, COCHRANE PLUS, LILACS, IBECS, WEB OF KNOWLEDGE, SCOPUS y OSH UPDATE.

Periodo de estudio: Publicaciones entre 2008 y 2012.

La búsqueda se estableció en base a Descriptores MeSH (DeCS):

"Occupational disease" AND "formaldehyde"

"Formaldehyde" AND "industry" AND "leukemia"

"Formaldehyde" AND "Leukemia"

"Formaldehyde" AND "Hematologic Neoplasm"

"Formaldehyde" AND "Mortality" AND "Leukemia"

\section{Criterios de inclusión:}

1. Estudios que describían la relación entre exposición laboral al formaldehído y la relación con leucemia.

2. Humanos trabajadores.

3. Año de publicación: entre 2008 y 2012. 
4. Artículos publicados en inglés y español.

5. Estudios descriptivos y analíticos.

\section{Criterios de exclusión:}

1. Estudios experimentales en animales.

2. Publicaciones no relevantes: Revisiones bibliográficas previas y cartas el editor.

3. Artículos redundantes.

4. Trabajos fuera del periodo de estudio.

Se obtuvo una colección de 302 artículos, de la cual se eliminaron los duplicados y se procedió a realizar un análisis de pertinencia revisando los títulos y los resúmenes de los artículos, quedando seleccionados 46 artículos a los que se les aplicaron los criterios de inclusión y exclusión, y obteniéndose finalmente un total de 7 artículos que conforman la base del estudio.

A los artículos seleccionados se aplicaron grados de evidencia científica mediante los criterios del Scottish Intercollegiate Guidelines Network (SING) ${ }^{9}$ descritos en la tabla II.

Tabla II. Criterios de Evidencia científica y grados de recomendación (SIGN)

\begin{tabular}{cl}
\hline Grado de evidencia & \multicolumn{1}{c}{ Criterios de evidencia } \\
\hline $1++$ & $\begin{array}{l}\text { Metaanálisis de gran calidad, revisiones sistemáticas de estudios aleatorizados y } \\
\text { controlados (EAC) o EAC de alta calidad y con muy bajo riesgo de sesgo. }\end{array}$ \\
Metaanálisis, revisiones sistemáticas de EAC o EAC bien desarrollados y con bajo \\
riesgo de sesgo. \\
Metaanálisis, revisiones sistemáticas de EAC o EAC bien desarrollados y con alto \\
riesgo de sesgo. \\
Revisiones sistemáticas de estudios de casos y controles o de estudios de \\
cohortes de alta calidad o estudios de casos y controles o de estudios de \\
cohortes con muy bajo riesgo de sesgo y con elevada probabilidad de que la \\
relación sea causal. \\
& $\begin{array}{l}\text { Estudios de casos y controles y estudios de cohortes bien desarrollados de } \\
\text { cohortes bien desarrollados, con bajo riesgo de sesgo y con probabilidad } \\
\text { moderada de que la relación sea causal. }\end{array}$ \\
& $\begin{array}{l}\text { Estudios de casos y controles, y estudios de cohortes con alto riesgo de sesgo y } \\
\text { con riesgo importante de que la relación no sea causal. }\end{array}$ \\
& Estudios no analíticos, como informes de casos y series de casos. \\
$2-$ & Opinión de expertos. \\
&
\end{tabular}

\section{RESULTADOS}

En la tabla III se presentan las principales características de los 7 artículos que componen el estudio, tales como: nombre del autor principal, título, descripción del diseño del estudio, tamaño de la población estudiada, resultados y conclusiones principales del estudio y el nivel de evidencia. 


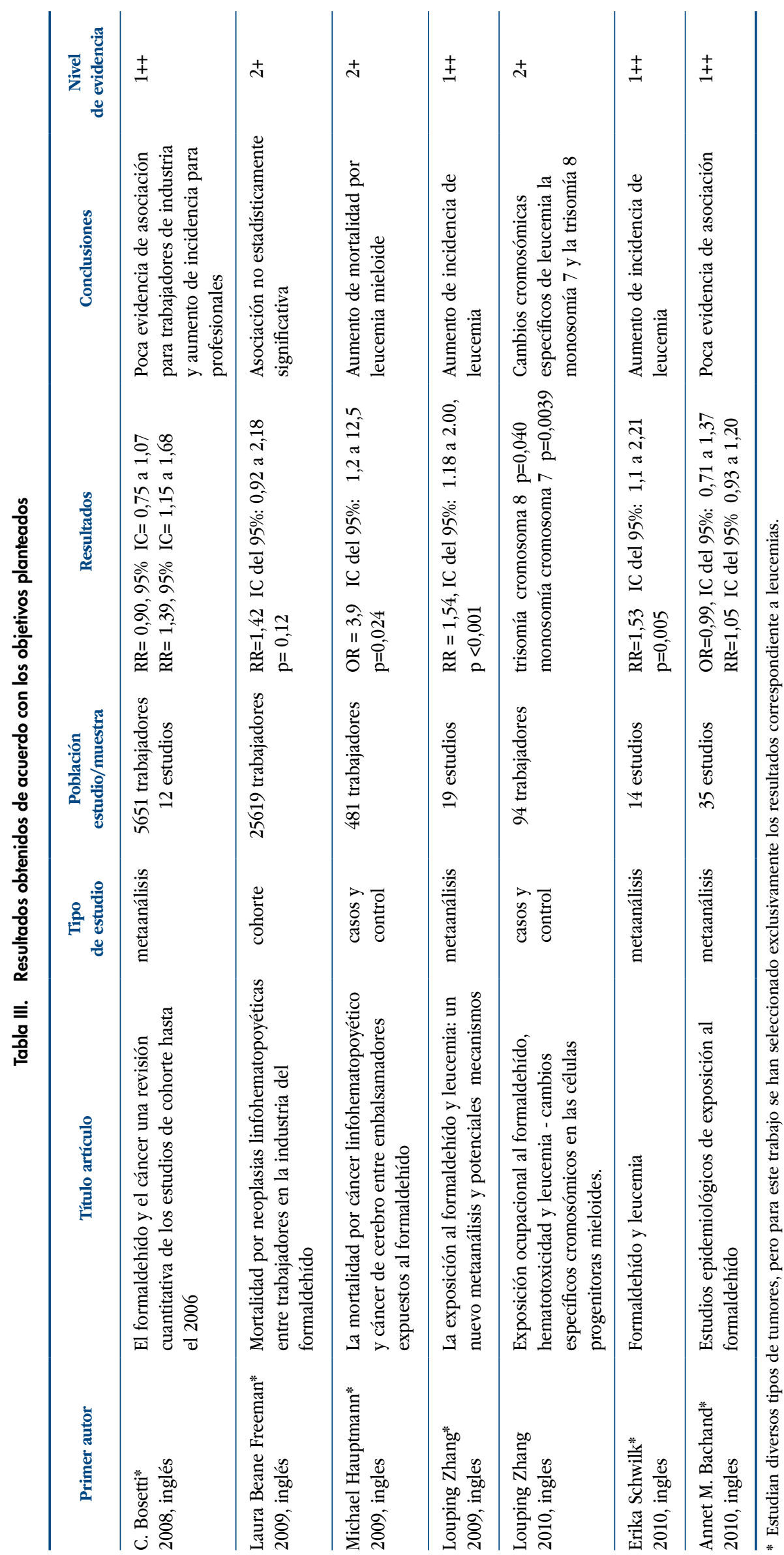


Los 7 artículos incluidos en la revisión presentan niveles de evidencia altos. De ellos, 4 son metaanálisis, cuyo nivel de evidencia se corresponde con 1+++, De los 3 restantes, 2 son estudios de casos y control y 1 de cohortes y estos 3 últimos presentan nivel de evidencia de 2+. Todos los artículos se publicaron entre 2008 y 2012.

A continuación se describen los principales resultados de cada uno de los artículos seleccionados.

Bossetti et al. ( 2008) ${ }^{10}$ publicaron una revisión cuantitativa de 16 cohortes originales publicadas hasta febrero del año 2007 que estudiaban la mortalidad por todos los tipos de cáncer en trabajadores profesionales (anatomistas, patólogos, forenses) y en trabajadores de la industria expuestos a formaldehído.

Entre sus resultados encontraron riesgos moderadamente elevados para los tumores linfohematopoyéticos en los profesionales pero no en los trabajadores de la industria.

Obtuvieron 234 muertes por todas las neoplasias hematopoyéticas y linfáticas en cuatro cohortes de trabajadores de la industria con un RR de 0,85.

En las cohortes de profesionales obtuvieron 263 muertes en ocho cohortes con significancia por un RR agrupado de 1,31 además siendo significativo el valor correspondiente a cinco cohortes de profesionales con estimaciones SMR de 1,27.

Con referencia a la leucemia obtuvieron 122 muertes en cuatro cohortes de trabajadores de la industria que corresponden a un RR agrupado de 0,90. En 8 cohortes de profesionales el RR combinado fue significativamente elevado (1,39) basado en 106 muertes.

Laura E Beane Freeman et al. (2009) ${ }^{11}$ presentaron una actualización de la cohorte del National Cancer Institute (NCI) ampliando el seguimiento hasta el año 2004. La cohorte incluyó a 25619 trabajadores seguidos durante un periodo de 42 años.

Mediante la regresión de Poisson calcularon el riesgo relativo (RR) y el 95\% de intervalos de confianza (IC) para examinar la asociación entre las estimaciones cuantitativas de exposición al formaldehído (exposición máxima, promedio de intensidad y exposición acumulada) y la muerte por enfermedades malignas linfohematopoyéticas. Además incluyeron en el análisis a 1006 muertes de trabajadores que ocurrieron entre 1980 y 1994 las cuales no habían sido incluidas en análisis previos.

Como resultado encontraron una asociación estadísticamente significativa entre neoplasias linfahematopoyéticas y la exposición máxima a formaldehido (tendencia de la $\mathrm{p}=0.02)(\mathrm{RR} 1,37$; IC $95 \%=1,03$ a 1,1$)$ entre aquellos trabajadores expuestos con exposiciones máximas mayores de $4,0 \mathrm{ppm}$ comparado con aquellos con exposiciones entre 0 y 2,0 ppm.

No encontraron asociación estadísticamente significativa para leucemia. El riesgo fué elevado en los máximas niveles de exposición (RR: 1,42; 95\% CI=0,92 a 2,18) y la tendencia de la $\mathrm{p}$ fue más baja cuando se incluyeron los no expuestos $(\mathrm{p}=0,02)$ que cuando no se incluyeron $(\mathrm{p}=0,12)$.

En el estudio de Michael Hauptmann et al. (2009) ${ }^{12}$ se investigó la relación entre la mortalidad por cáncer linfohematopoyético y tumores cerebrales entre embalsamadores expuestos a formaldehído.

Consistió en un estudio de casos y controles con 216 trabajadores fallecidos a causa de enfermedades malignas linfohematopoyéticas y tumores cerebrales que fueron comparados con 265 sujetos control.

La mortalidad por la leucemia mieloide aumentó de manera estadísticamente significativa con el aumento del número de años de realización de embalsamamientos $(\mathrm{p}=0,020)$ y con el aumento de exposición máxima $(\mathrm{p}=0,036)$.

Encontraron que la mortalidad por leucemia mieloide fue elevada entre aquellos que realizaron embalsamamientos durante más de 34 años $(\mathrm{OR}=3.9,95 \% \mathrm{CI}=1.2$ a 1.5 , 
$\mathrm{p}=.024)$ y entre quienes realizaron más de 3068 embalsamamientos $(\mathrm{OR}=3.0,95 \% \mathrm{CI}=1.0$ a 9.2, $\mathrm{P}=0.057)$ al igual que en aquellos cuya exposición acumulada superó 9253 partes por millón-horas (OR $=3,1$, IC 95\% = 1,0 a 9,6, P = 0,047) en comparación con sujetos que realizaron menos de 500 embalsamamientos.

En cuanto a las limitaciones, el estudio no contó con una población de base (se utilizaron certificados de defunción) y datos obtenidos en entrevistas con familiares y compañeros de trabajo.

Zhang et al. (2009) ${ }^{13}$ presentaron un metaanálisis en donde se utilizaron datos de 19 estudios. El riesgo relativo (RR) para todos los tipos de cánceres linfohematopoyéticos fue de 1,25 (IC del 95\%, 1.09-1.43), para todas las leucemias (RR = 1,54, 95\% CI, 1.18-2.00, $\mathrm{p}<0,001)$ y para la leucemia mieloide $(\mathrm{RR}=1,90, \mathrm{IC} 95 \%, 1.31-2.76, \mathrm{p}=0,001)$.

Mediante la metodología de selección de datos sobre los grupos más expuestos de cada estudio se obtuvieron riesgos relativos altos que proporcionaron evidencia principalmente para leucemia mieloide.

Zhang et al. (2010) ${ }^{14}$ estudiaron si la exposición al formaldehído altera la función hematopoyética y produce leucemia relacionada con cambios en los cromosomas en trabajadores expuestos mediante un estudio de casos-control con 94 trabajadores en China de los cuales 43 fueron casos expuestos y 51 controles.

En los casos expuestos se estableció que no había otras exposiciones hematotóxicas como benceno, fenol y disolventes clorados. Los controles se seleccionaron a partir de tres lugares de trabajo en la misma región geográfica sin la exposición al formaldehído ni otros hematotóxicos.

Entre los trabajadores expuestos los recuentos de células sanguíneas periféricas fueron significativamente más bajos de una manera consistente con los efectos tóxicos sobre la medula ósea y los cambios cromosómicos específicos de leucemia como la monosomía 7 y la trisomía 8 . Con resultado de $\mathrm{p}=0.040$ para la trisomía 8 y resultado $\mathrm{p}=0.0039$ para la monosomía 7 .

Erika Schwilk et al. (2010) ${ }^{15}$ realizaron un meta-análisis para evaluar la relación entre la exposición al formaldehído y la leucemia.

Este metaanálisis se basó en 14 estudios (13 de cohortes y 1 estudio de casos y control ) de los cuales 6 estudios fueron de profesionales del ámbito sanitario y 8 estudios de grupos industriales. Se destacan dos estudios: uno que involucró a 25.000 trabajadores en industrias de formaldehído en EEUU con 40 años de seguimiento y otro de casos y control realizado en $\mathbf{1 3 . 0 0 0}$ trabajadores del sector funerario.

El formaldehído se asoció con un mayor riesgo de leucemia ( $R R=1.53$, IC del 95\% de 1.11 a $2.21, \mathrm{P}=0.005)$ y de leucemia mieloide $(\mathrm{RR}=2.47$, IC del $95 \%$ de 1.42 a $4.27, \mathrm{P}=0.001)$.

En este metaanálisis el RR fue mayor para los trabajadores profesionales que para los trabajadores de la industria aunque en estos los hallazgos no fueron negativos.

Annette M.Bachand et al. (2010) ${ }^{11}$ realizaron un metaanálisis donde resumieron los resultados de estudios de cohortes y de casos control hasta mayo del 2009 y se excluyeron los estudios PMR (estudios de mortalidad proporcional), que son metodológicamente limitados y difíciles de interpretar. Se realizaron análisis de sensibilidad para investigar el efecto de ajuste por tabaquismo y otros factores de confusión y utilizaron cuantiles y modelos de regresión para evaluar la heterogeneidad y el posible sesgo de publicación.

Para leucemias el RR fue 1,05, IC del 95\% de 0,93 a 1,20 para los estudios de cohortes y la OR fue de 0,99 , IC 95\% de 0,71 a 1,37 para los estudios de casos y controles.

Este metaanálisis encuentra poca evidencia de asociación entre la exposición al formaldehído y leucemia. Además atribuye que el mayor riesgo de asociación visto en estudios anteriores, se debe a que incluyeron los estudios PMR en su análisis. 
En conclusión cuatro de los 7 estudios incluidos en esta revisión aportan resultados que apoyan a la evidencia existente de nuestro objetivo de estudio.

\section{DISCUSIÓN}

Estudios previos han encontrado una asociación positiva entre la exposición a formaldehido y leucemia: Blair et al. (1990), Hauptmann et al. (2003), Pinkerton et al. (2004), Collins y Lineker (2004), aunque otros estudios no encontraron ninguna asociación (Coggon et al., 2003) ${ }^{17}$.

En estudios más recientes, coincidiendo con nuestra búsqueda encontramos una variedad de estudios con gran heterogeneidad por lo cual la evidencia de la asociación de formaldehído y leucemia continua siendo causa de análisis.

Michael Hauptmann (2009) ${ }^{12}$ halla un aumento de la mortalidad en relación a la duración de la práctica de embalsamamiento. Autores como, Robet Golden ${ }^{18}$ considera $^{2}$ que es un estudio caso control incrustado en un estudio de mortalidad proporcional donde no hay mediciones del formaldehído porque no estaban disponibles y se han realizado estimaciones basadas en modelos de exposición. Golden ${ }^{18}$ resalta que el estudio de Hauptmann ${ }^{12}$ no tiene en cuenta el efecto de otras sustancias químicas con carácter mutagénico utilizadas frecuentemente en las prácticas de embalsamamiento que podrían influenciar estos resultados, además de que no tiene en cuenta las variaciones en las técnicas de realización de embalsamamientos.

Laura Freeman ${ }^{11}$ actualiza la cohorte del National Cancer Institute y aporta también RR elevados para todas las enfermedades linfahematopoyeticas, incluida leucemia, teniendo en cuenta los valores de máxima exposición al formaldehído. Así mismo Zhang et al (2009) ${ }^{13}$ examina grupos de mayor exposición y aporta el mayor riesgo relativo visto en los estudios de leucemia mieloide. Bachand et al. (2010) ${ }^{11}$ se basa en niveles de exposición menos altas al formaldehído y al excluir los estudios de tasas de mortalidad proporcional (PMR) obtiene poca evidencia constituyendo el único estudio de ésta revisión en contra de una relación de asociación entre formaldehído y leucemia.

Golden $^{18}$ en su trabajo de revisión discrepa de los resultados obtenidos por Zhang (2009) ${ }^{12}$ en cuanto a que no es posible determinar si el RR ha sido mayor en las cohortes más expuestas o al menos hacer una comparación entre las cohortes más expuestas como lo realizan los dos estudios de Collins y Lineker (2004) ${ }^{17}$ y Bachand (2010) ${ }^{11}$ quienes estratifican y analizan los datos por separado en función de los trabajadores con baja exposición (embalsamadores, anatomistas) y de alta exposición (trabajadores industriales).

Erika Schwilk ${ }^{15}$ et al. coinciden con Coggon et al., al encontrar valores significativos con $\mathrm{p}<0,05$ y con un RR mayor para los trabajadores profesionales (anatomistas, patólogos, forenses, etc.). También Bosetti et al (2008) ${ }^{10}$ encuentra RR elevados para mortalidad por leucemia en trabajadores profesionales.

Al igual que Zhang et al. (2010) ${ }^{14}$, algunos estudios sobre los efectos cromosómicos de personas expuestas a fomaldehído intentan explicar la base del mecanismo genotóxico: el estudio de Mátyás G. Jakaba et al. ${ }^{19}$ encuentra que en una pequeña población de mujeres expuestas a formaldehído en laboratorios de anatomía patológica en Hungría presentaron cambios cromosómicos específicos de leucemia tales como cambios apoptóticos y aberraciones cromosómicas en linfocitos en sangre periférica que sugieren datos de asociación entre la exposición al formaldehído y leucemia in vivo. Aunque es posible que para encontrar el nivel de evidencia se requieran estudios adicionales preferiblemente con un mayor tamaño muestral.

Zhang et al. (2009) ${ }^{13}$ en su metaanálisis propone tres posibles mecanismos patogénicos en la inducción de leucemia: el primero por lesión directa del formaldehído a nivel de células madre en la médula ósea como lo hacen la mayoría de las leucemógenos. El segundo mecanismo por lesión de células madre progenitoras presentes en la sangre 
circulante y el tercero por lesión directa de células madre pluripotenciales primitivas presentes en el tejido de mucosa olfativa y cornetes nasales.

En el primer mecanismo Zhang et al. ${ }^{13}$, afirma que el formaldehído potencialmente podría llegar a la médula ósea en forma de methanediol (que es una forma hidratada el formaldehído) através de la circulación sanguínea y causar lesión directa del ADN y aberraciones cromosómicas en células madre hematopoyéticas promoviendo el desarrollo de las células madre leucémicas. Autores como Bernard Goldstein ${ }^{20}$ apoyan este mecanismo, pero comentan que sería necesario más estudios para determinar si el methanediol es un compuesto que pueda persistir en la circulación hasta llegar a la medula ósea y producir estos cambios.

De acuerdo al segundo mecanismo propuesto por Zhang et al. ${ }^{13}$ el formaldehído podría causar leucemia una vez que alcanza la circulación sanguínea e induce mutaciones o lesiones premutagénicas en las células madre hematopoyéticas circulantes, Bernard Goldstein $^{20}$ afirma que las dosis las sustancias leucemógenas que producen leucemia mieloide aguda producen también pancitopenia, lo que no ha sido fácilmente observable con la exposición a formaldehído a pesar de unos amplios estudios en animales y humanos.

Para el tercer mecanismo que propone Zhang et al. ${ }^{13}$, que implicaría lesiones premutagénicas en células madre de la mucosa nasal, Goldstein ${ }^{20}$ afirma que se podría aplicar la misma hipótesis en el caso del Cromo que es un carcinógeno nasal conocido, pero se ha demostrado en varios estudios que la mortalidad por leucemia es muy escasa en trabajadores que utilizan el Cromo.

A pesar de las limitaciones, los estudios biológicos de este tipo proporcionan resultados positivos indicando que el formaldehído puede causar una serie de efectos genotóxicos en el ADN y los cromosomas de los linfocitos así como en las células derivadas de la médula ósea.

En el futuro el uso de biomarcadores de dosis interna acumulada tales como aductos ADN-formaldehído, aductos entre el glutatión y la lesión del ADN inducida por formaldehído podrán mejorar el desarrollo de estos estudios

Teniendo en cuenta los hallazgos hasta el momento la Enviromental Protection Agency (EPA) de los Estados Unidos ha publicado que el formaldehído es cancerígeno cuando es inhalado por los seres humanos. "Hay pruebas suficientes de una relación causal entre la exposición al formaldehído y el cáncer de las vías respiratorias superiores, con la evidencia más fuerte para el cáncer nasofaríngeo y sinunasal". Además en junio de 2011 publica que "También hay pruebas suficientes de una asociación causal entre la exposición al formaldehído y el cáncer linfohematopoyético, con mayor evidencia de linfoma de Hodking y leucemia, especialmente leucemia mieloide" ${ }^{21}$.

\section{CONCLUSIONES}

La agencia internacional del cáncer (IARC) en el año 2004 reclasifica al formaldehído como un cancerígeno humano y en el año 2006 concluye que hay suficiente información para relacionar la exposición al formaldehído y la leucemia en particular con la leucemia mieloide.

Esta revisión contiene estudios heterogéneos realizados en diferentes poblaciones de trabajadores expuestos a formaldehído, algunos de ellos encuentran valores de asociación (RR, OR) superiores a 1 para riesgos elevados en niveles de máxima exposición y también datos de mortalidad estadísticamente significativa para leucemia mieloide.

Los resultados de dos estudios que incluyen pacientes de la cohorte del National Cancer Institute actualizada por Freeman y el estudio de casos de control de Hauptmann muestran datos de asociación estadística con las altas exposiciones al formaldehído pero 
presentan limitaciones que aún no permiten un consenso para extrapolar estos resultados a la población trabajadora expuesta.

Como ejemplos de las limitaciones de los estudios de nuestra revisión están que las estimaciones de exposición a formaldehído estaban basadas en modelos de exposición sin corresponder a mediciones reales, la posible influencia de la exposición a otras sustancias químicas en los lugares de trabajo y los factores inherentes a los trabajadores tales como la susceptibilidad genética, el antecedente de consumo de tabaco entre otros.

Junto con los datos de asociación significativa entre la exposición a formaldehído y riesgo de leucemia aportada por algunos de estos estudios, se plantean también las alteraciones cromosómicas encontradas en personas expuestas, ambos constituyen una base interesante para investigaciones futuras donde la utilización de biomarcadores de dósis interna acumulada (Aductos ADN-Formaldehído, Glutatión-lesión de ADN inducida por formaldehído) podrán verificar mejor esta asociación.

El formaldehído es una sustancia ampliamente utilizada en nuestro medio a nivel industrial y en el ámbito sanitario, Por tal motivo se hace necesario proteger el personal expuesto de sus efectos tóxicos, alérgicos y cancerígenos aplicando formación e información preventiva a las personas que utilizan este compuesto en sus lugares de trabajo.

\section{REFERENCIAS BIBLIOGRÁFICAS}

1. Lauwerys RR. Toxicología industrial e intoxicaciones profesionales, Masson, editor , 1994 , pág. 346.

2. Pascual del Rio J. Agentes químicos en el ámbito sanitario , Escuela Nacional de Medicina del Trabajo (ENMT). Madrid 2010. Capítulo 4, págs. 161-186.

3. European Federation of Buildind and Woodworkers, Proyecto Social Ref-Wood, Reducción de la exposición al formaldehído en la industria de la madera (citado 2012 diciembre 19) Disponible en : http:// www.cei-bois.org/files/Brochure_Spanish.pdf.

4. International Agency for Research on Cancer. La OMS considera cancerígeno el formaldehído. Revista Española de Patología 2005; 38(1) 62-63.

5. International Agency for Research on Cancer. IARC Press Realece No 153; 2004 (citado 2012 diciembre 12). Disponible en: http://www.iarc.fr/en/media-centre/pr/2004/pr153.html http://monographs.iarc.fr/ ENG/Classification/Table4.pdf.

6. Instituto Nacional de Seguridad e Higiene en el Trabajo (citado 2013 enero 19). Disponible en: http:// bdlep.insht.es/LEP2012.

7. Grupo Colaborativo del Registro Español de Leucemias. Resultados del Registro Español de Leucemias (REL) 2002, pág. 15 (citado 2013 diciembre 20). Disponible en http://www.leucemiaylinfoma.com/web_ old/proyectos/pdf/MAQ.01-REL\%202002.pdf.

8. Zhang L, Freeman LE, Nakamura J, Hecht SS, Vandenberg JJ, Smith MT. Formaldehyde and leukemia: epidemiology, potential mechanisms, and implications for risk assessment. Environmental and Molecular Mutagenesis. 2010 Apr; 51(3):181-91.

9. Scottish Intercollegiate Guidelines Network (SIGN). SIGN 50, A guideline developer's handbook, First published 2008, Revised November 2011. Disponible en http://www.sign.ac.uk/pdf/sign50.pdf.

10. Bosetti C, McLaughlin JK, Tarone RE, Pira E, La Vecchia C. Formaldehyde and cancer risk: a quantitative review of cohort studies through 2006. Annals of Oncology 2008; 19: 29-43 (citado 2013 enero 10). Disponible en: http://annonc.oxfordjournals.org/content/19/1/29.full.

11. Beane Freeman LE, Blair A, Lubin JH, Stewart PA, Hayes RB, Hoover RN. Mortality From Lymphohematopoietic Malignancies Among Workers in Formaldehyde Industries: The National Cancer Institute Cohort. Journal of the National Cancer Institute. 2009 May 20; 101(10): 751-761.

12. Hauptmann M, Stewart PA, Lubin JH, Beane Freeman LE, Hornung RW, Herrick RF. Mortality from lymphohematopoietic malignancies and brain cancer among embalmers exposed to formaldehyde. Journal of the National Cancer Institute 2009 Dec 16; 101(24): 1696-708.

13. Zhang L, Steinmaus C, Eastmond DA, Xin XK, Smith MT. Formaldehyde exposure and leukemia: a new meta-analysis and potential mechanisms. Mutation Research. 2009 Mar-Jun; 681(2-3): 150-68. 
14. Zhang L, Tang X, Rothman N, Vermeulen R, Ji Z, Shen M. Occupational exposure to formaldehyde, hematotoxicity, and leukemia-specific chromosome changes in cultured myeloid progenitor cells. Cancer Epidemiology Biomarkers Prevention. 2010 Jan; 19(1): 80-8

15. Schwilk E, Zhang L, Smith MT, Smith AH, Steinmaus C. Formaldehyde and leukemia: an updated metaanalysis and evaluation of bias. Journal of Occupational and Environmental Medicine. 2010 Sep; 52(9): 878-86.

16. Bachand AM, Mundt KA, Mundt DJ, Montgomery RR. Epidemiological studies of formaldehyde exposure and risk of leukemia and nasopharyngeal cancer: a meta-analysis. Critical Reviews in Toxicology. 2010; 40(2): 85-100.

17. Collins J, Lineker G. A review and metaanalysis of formaldehyde exposure and leukemia. Regulatory Toxicology and Pharmacology 2004; 40: 81-91.

18. Golden R. Identifying an indoor air exposure limit for formaldehyde considering both irritation and cancer hazards. Critical Reviews in Toxicology 2011 Sep; 41(8): 672-721.

19. Matyas GJ, Klupp T, Besenyei C, Biró A, Major J. Formaldehyde induced chromosomal aberrations and apopstosis in peripheral blood lymphocites of personnel working in pathology departments. Mutation Research 2010; 698: 11-17.

20. Goldstein BD. Hematological and toxicological evaluation of formaldehyde as a potential cause of human leukemia. Human Experimental Toxicology. 2011 Jul; 30(7): 725-35.

21. IRIS Toxicological Review of Formaldehyde (Inhalation) (External Review Draft 2010). Enviromental Protection Agency de los Estados Unidos (citado 2013 enero 19). Disponible en http://cfpub.epa.gov/ ncea/iris_drafts/recordisplay.cfm?deid=223614.

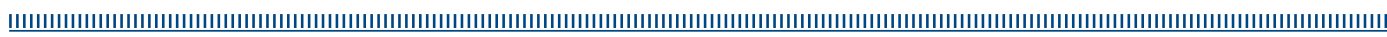

